\title{
Validación de la encuesta de clima organizacional de Pulido en colaboradores de tres empresas de Lima Metropolitana.
}

Validation of the organizational climate survey's Pulido in three companies of Lima Metropolitan.

\author{
Ruth Polett Pulido Ramirez ${ }^{1}$
}

\section{RESUMEN}

El propósito de la investigación fue la de validar la Encuesta de Clima Organizacional de Pulido (ECOP) a fin de aportar evidencias sobre la utilidad de este instrumento en el ámbito organizacional. La encuesta fue aplicada a 1285 colaboradores de tres empresas. Luego, se realizó un análisis de base de datos secundaria con la finalidad de validar el instrumento por medio de un análisis factorial confirmatorio (AFC) basado en los modelos de ecuaciones estructurales y de esa forma complementar la información con la que cuenta en la actualidad y que fue obtenida por medio del análisis factorial exploratorio. Se logró obtener una correcta validez de constructo en base a tres factores y no de cuatro factores como se había postulado en la encuesta original.

PALABRAS CLAVES: Clima organizacional, encuesta de Pulido, factorial confirmatorio.

\section{SUMMARY}

The purpose of the research was to validate the Polished Organizational Climate Survey (ECOP) in order to provide evidence on the usefulness of this instrument in the organizational field. The survey was applied to 1285 employees of three companies. Second, a secondary database analysis was carried out with the purpose of validating the instrument through a confirmatory factor analysis (AFC) based on the structural equation models and thus complementing the information that it currently has and Which was obtained by exploratory factor analysis. It was possible to obtain a correct validity of construct based on three factors and not of four factors as had been postulated in the original survey.

KEYWORDS: Organizational climate, survey's Pulido, confirming factorial.

\section{INTRODUCCIÓN}

\section{Validez de constructos hipotéticos en Psicología}

Esta investigación constituyó un intento de dar respuesta a múltiples interrogantes que surgen cuando el psicólogo alterna su cotidiano rol de usuario de la ciencia y la tecnología psicológica en el plano de la prestación de servicios o de la enseñanza, por el rol de observador de las teorías psicológicas que son el sustento de tests y otros instrumentos de medición y evaluación en este campo. Tal alternancia no es práctica frecuente y requiere, además de una motivación enraizada en la Filosofía de la Ciencia, tomar distancia para examinar en perspectiva los supuestos que subyacen a las teorías psicológicas, a modo de axiomas de un sistema lógico-axiomático y de términos primitivos anteriores al sistema y fuera de él (Miljánovich, 1997). 
En este examen destacan los constructos hipotéticos como una clase importante de elementos que juegan un papel decisivo en la significatividad cognoscitiva de las observaciones, en tanto éstas constituyen observaciones de clases de fenómenos y, en cuanto se relacionan entre sí, en términos hipotéticos, conjuntos de observaciones, dentro del marco de una teoría, que inicialmente aporta dicho marco y que al propio tiempo, por retroinformación, tiende a crecer y a sistematizarse (Miljánovich, 1997).

Según Nunnally y Berenstein (1995), hay tres aspectos importantes en la validación de un constructo a saber: 1) especificar el dominio de variables observables relacionado con el constructo, 2) determinar el grado en que las variables observables tienden a medir lo mismo, varias cosas diferentes o muchas cosas diferentes por medio de investigación empírica y análisis estadísticos, y 3) realizar estudios subsecuentes $\mathrm{y} / \mathrm{o}$ experimentos de diferencias individuales para determinar el grado en que las supuestas mediciones del constructo son consistentes con las "mejores conjeturas" acerca del constructo (Miljánovich, 1997). El aspecto 3) consiste en determinar si una supuesta medida de un constructo se correlaciona en las formas esperadas con las medidas de otros constructos y/o es afectado en los modos esperados por la manipulaciones experimentales apropiadas (Miljánovich, 1997). Con frecuencia, un constructo en particular se vuelve popular y diferentes investigadores intentar concebir sus propias medidas. Conforme crece la cantidad de medidas propuestas del constructo, crece la sospecha de que podría ser que no todas midan lo mismo. Uno o más investigadores buscar delimitar el dominio de variables observables relacionadas con el constructo (aspecto 1). Toda la delimitación o parte de ella es sometida a investigación para determinar el grado en que estas medidas alternativas son o no equivalentes (aspecto 2). El impacto de la teorización del aspecto 1 y los resultados de la investigación del aspecto 2 tienden a influir en la determinación de cuales variables particulares se van a estudiar (aspecto 3) (Miljánovich, 1997). Cuando este proceso complejo cubre secuencialmente los tres aspectos descritos, tenemos como resultado un constructo que: 1) está bien definido a través de una variedad de observables, 2) está bien representado por medidas alternativas y 3 ) se relaciona fuertemente con otros constructos de interés (Miljánovich, 1997).

Miljánovich (1997) concluye finalmente que la validación de constructos hipotéticos en psicología involucra el proceso de hacer explícita una palabra o una frase abstracta en términos de variables observables. En este esfuerzo hay a menudo un riesgo potencial, que es el tratar la palabra o frase como si denotara una entidad o proceso real, esto es, materializando tal denotación. Dicho riesgo se pone en evidencia cuando un psicólogo dice "esto no es en realidad una medida de la ansiedad". En la expresión se encuentra el supuesto implícito de que el constructo "ansiedad" tiene una realidad objetiva, es decir, que existe más allá de las medidas propuestas para describirlos. Lo deseable en este contexto es usar el término "ansiedad" como una denominación conveniente para un conjunto particular de variables observables, cuya validez reside solo en el grado en que describa con precisión los tipos de variables observables propios de su dominio.

\section{Validez factorial de la encuesta de clima laboral de Ricardo Valenzuela}

El propósito del estudio fue proporcionar una herramienta para la medición del clima organizacional, la cual sirvió de apoyo en la toma de decisiones y ha sido aplicada durante procesos de cambio organizacional para mejorar la efectividad de una organización (Salaiza, 2008). El instrumento que se validó, consta de dos partes: la primera plantea tres preguntas de respuesta abierta y la segunda que incluye un cuestionario de 80 preguntas de respuesta cerrada, tipo Likert de cinco puntos (uno es la percepción más negativa y cinco la más positiva) sobre el clima laboral agrupadas en diez escalas o categorías: trabajo personal, supervisión, trabajo en equipo y relaciones con compañeros de trabajo, administración, comunicación, ambiente físico y cultural, capacitación y desarrollo, promoción y carrera, sueldos y prestaciones y orgullo de pertenencia (Salaiza, 2008). Se realizaron tres tipos de análisis estadísticos (Salaiza, 2008): a) Análisis estadístico descriptivo: este análisis implicó determinar medidas de tendencia central, medidas de dispersión y medidas de riesgo; b) Análisis de los instrumentos: consistió en evaluar la calidad del instrumento. Esto implicó el determinar indicadores de confiabilidad; consistencia interna, test-restest y se realizaron tres tipos de validez al instrumento: -Validez de contenido: se obtuvo mediante la comparación del contenido del instrumento con el universo de contenidos y conductas a ser medidas. Si un test tiene validez de contenido, se puede decir que sus calificaciones son eficaces (Valenzuela, 2005): Validez de criterio, consistió en qué tan acertadamente el test permite predecir el desempeño futuro o estimar el desempeño actual de una medición diferente de la del test en cuestión y validez de constructo, referido 
a la forma de interpretar la ejecución de una prueba en términos de alguna característica, cualidad o constructo psicológico. Se realizó mediante el análisis factorial exploratorio y c) La interpretación conceptual, que consistió en buscar relaciones entre las variables medidas e interpretar el significado de dichas relaciones en términos del marco teórico. Fue una forma de profundizar en la comprensión que se tiene del constructo y lograr determinar el grado en el que dicho constructo puede ser medido adecuadamente con el instrumento de clima laboral.

\section{Análisis psicométrico de la encuesta ECO IV de clima organizacional por países}

El propósito de esta investigación fue de realizar una primera exploración sistemática del comportamiento psicométrico de la encuesta ECO IV de clima organizacional en varios países de América Latina. En ocasiones precedentes se han llevado a cabo verificaciones asiladas en algunos países (Cincel, 2007), con resultados bastante positivos. En este estudio se realizó una exploración sistemática y comparativa con datos obtenidos en ocho países de la región: Colombia, Venezuela, Ecuador, México, Costa Rica, Perú, Estados Unidos y Panamá (Toro, 2008). La encuesta ECO IV de clima organizacional está conformada por un conjunto de reactivos que deben ser respondidas en una escala de tipo Likert de cuatro puntos, con extremos entre uno (completamente en desacuerdo) y cuatro (completamente de acuerdo). Identifica las percepciones del personal en relación con diez realidades importantes del trabajo en la empresa (Toro, 2001). Se trata de un instrumento psicométrico factorial que, a través de 63 ítems, evalúa diez variables de clima organizacional. El estudio más reciente de validación realizado en Colombia en enero de 2008, mostró un nivel de confiabilidad de .96 obtenido mediante el método Alfa de Cronbach y de .95 con el método de correlación de dos mitades (Cincel, 2008). Para este cometido se llevaron a cabo varios análisis con fundamento en la Teoría Psicométrica Clásica. Se examinó, el funcionamiento individual de cada uno de los ítems a través de la determinación de su capacidad de discriminación, evaluada mediante cálculo del promedio de las respuestas dadas por la muestra y de su dispersión (desviación estándar), considerando que será mejor su discriminación si su promedio se encuentra cerca del punto medio de la escala (2.5) y su dispersión es amplia (superior a 1.0, en este caso) (Toro, 2007; Mercado y Toro, 2008). También exploró la correlación ítem-test, que es una medida adicional de calidad que se obtiene a través del cálculo de un coeficiente de correlación. En relación con la confiabilidad de la encuesta se obtuvo su coeficiente por dos métodos: Alfa de Crombach y Dos Mitades (Nunnally y Bernstein, 1995). En relación con su validez de construcción se aplicó el análisis factorial confirmatorio con rotación Varimax (Grimm y Yarnold, 1998). Este análisis buscó poner en evidencia la existencia y consistencia de cada variable y de sus componentes a través de las muestras de los distintos países (Toro, 2008).

\section{Diseño y validación de un modelo de medición del clima organizacional basado en percepciones $y$ expectativas.}

El diagnóstico del clima organizacional es una herramienta de gestión organizacional de las percepciones que tienen los miembros de una organización respecto del ambiente en que desarrollan sus actividades laborales. En este estudio, se propuso un modelo de diagnóstico del clima organizacional basado en medir, además de las percepciones, las expectativas que tienen los individuos respecto del ambiente en que se desenvuelven. En paralelo se desarrolló y validó un instrumento de medición que estima los niveles de percepciones y de expectativas para construir un indicador de clima organizacional a partir de la brechas entre ambos. El instrumento aportó información relevante para la toma de decisiones en gestión organizacional, ya que permite identificar las brechas percibidas por los miembros de la organización respecto del ambiente interno en que se desarrollan las actividades, permitiendo una focalización adecuada de las acciones de mejora en este aspecto (Reinoso y Araneda, 2007).

A partir del estudio de la teoría de clima organizacional clásica y la realización de entrevistas con psicólogos organizacionales, se adoptan las dimensiones del clima organizacional que aparecen con mayor frecuencia en los estudios, y en torno a éstas se construyó un instrumento que mide las brechas existentes entre las percepciones y expectativas de los individuos para cada una de ellas. Se realizó una aplicación piloto, que permitió realizar el análisis de confiabilidad por medio del Alfa de Cronbach, ampliamente reconocido en la investigación en administración. Posteriormente, se realizan los análisis de validez de contenido, criterio y constructo, utilizando las herramientas estadísticas de correlación entre variables de Pearsons (Reinoso y Araneda, 2007). 


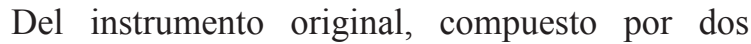

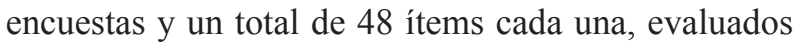

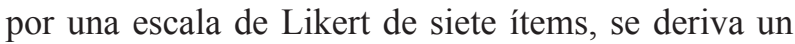

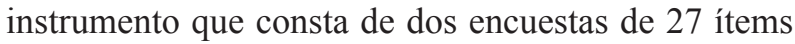

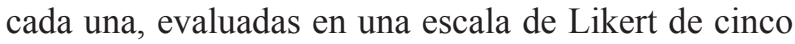

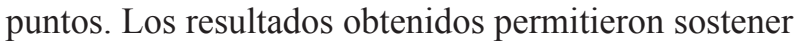

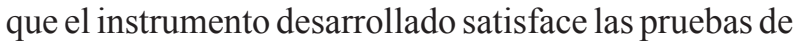

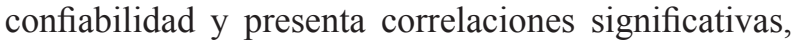

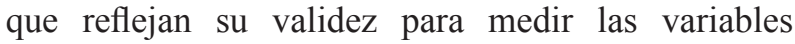

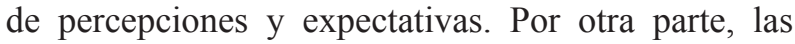

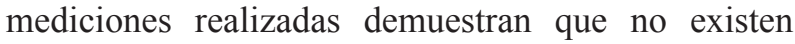

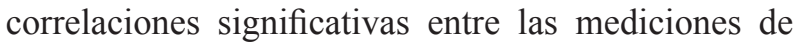

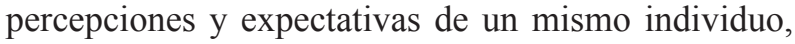

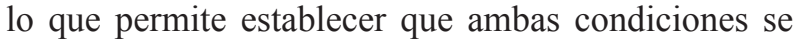

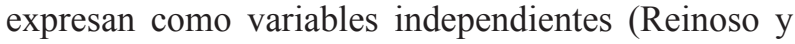

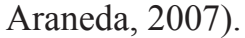

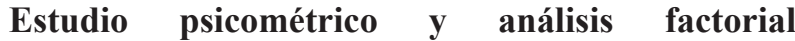

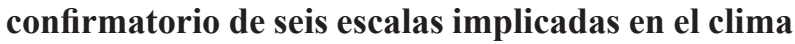

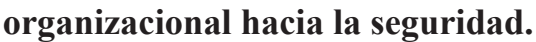

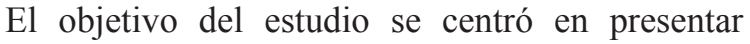

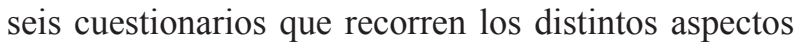

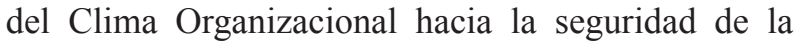

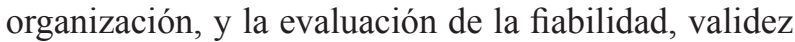

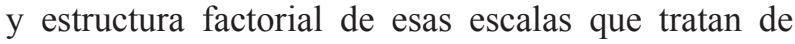

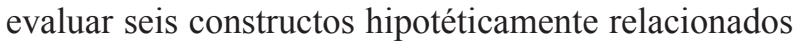

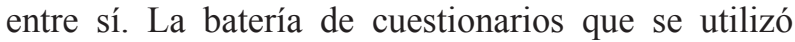

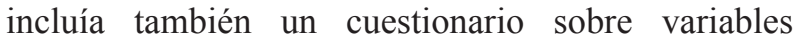

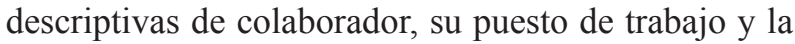

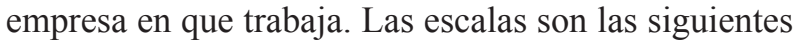

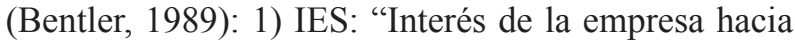

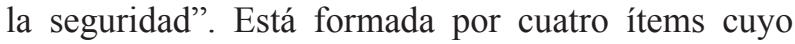

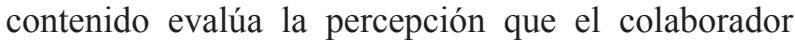

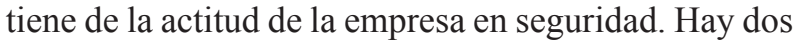

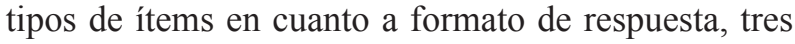

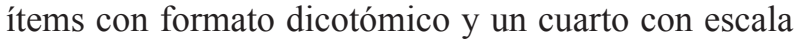

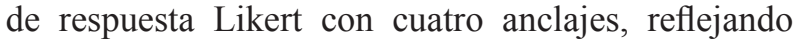

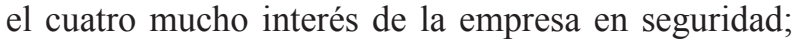

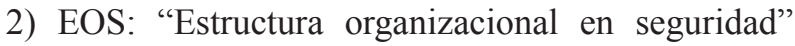

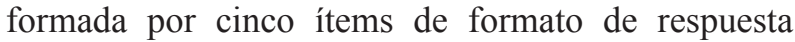

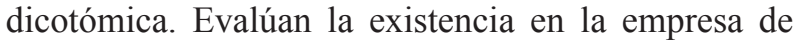

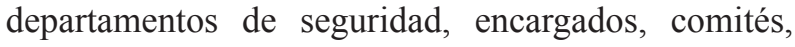

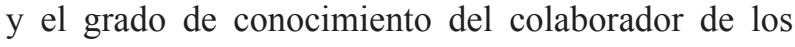

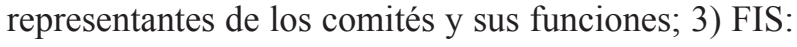

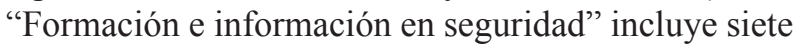

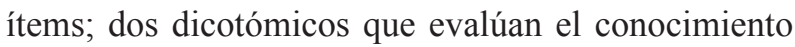

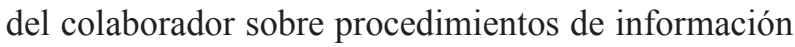

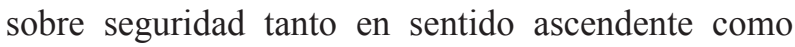

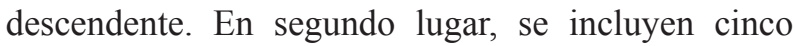

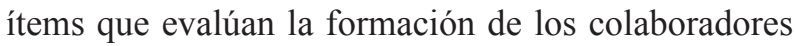

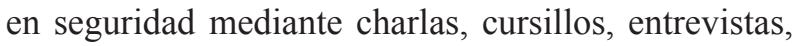

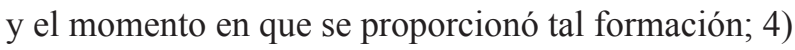

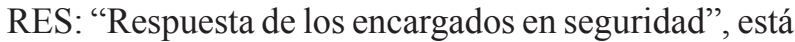

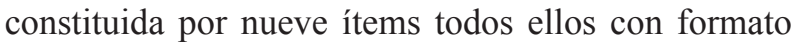

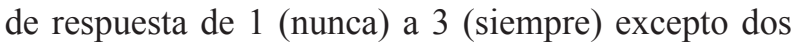

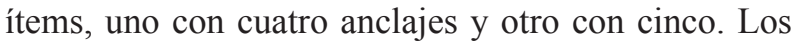

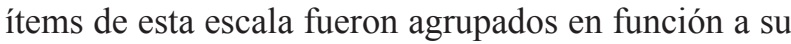

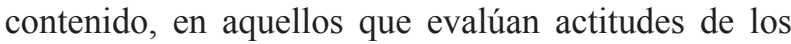

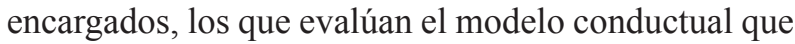

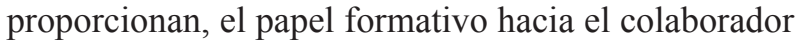

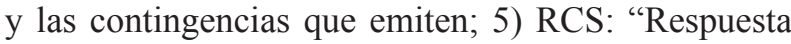

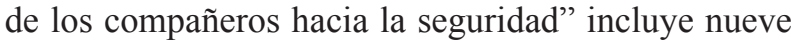

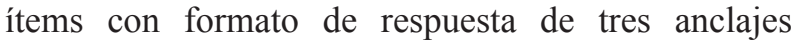

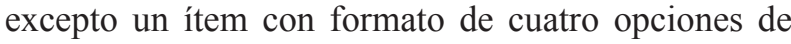

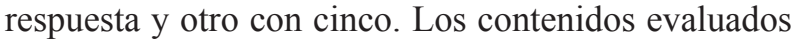

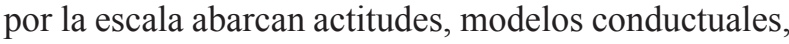

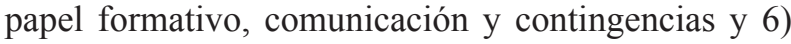

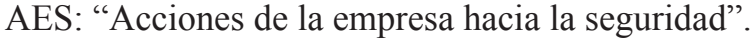

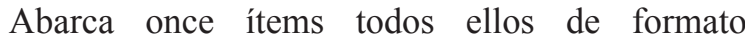

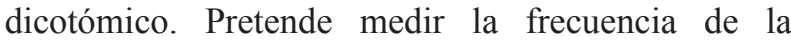

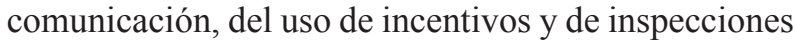

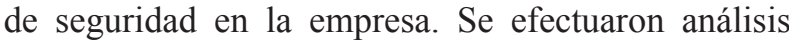

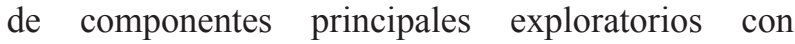

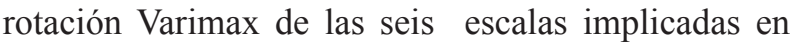

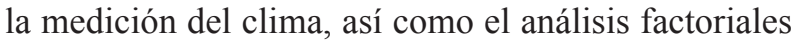

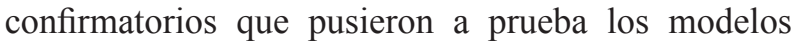

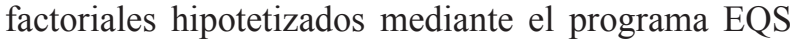

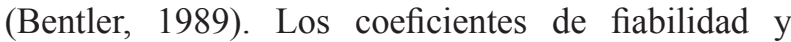

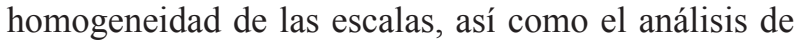

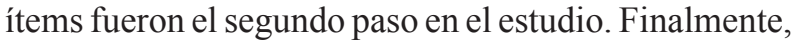

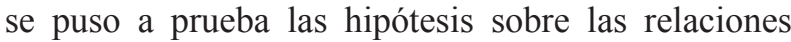

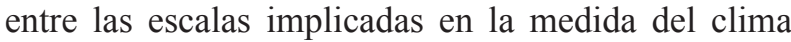

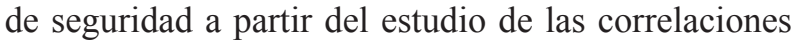

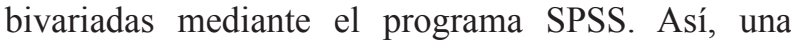

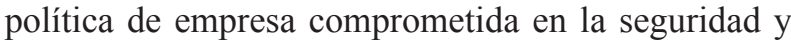

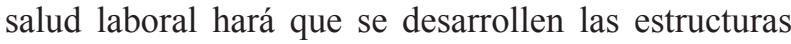

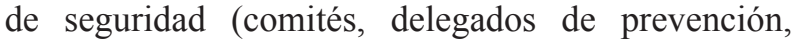

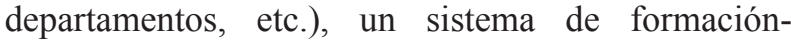

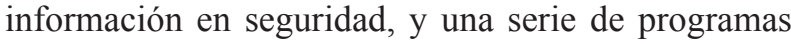

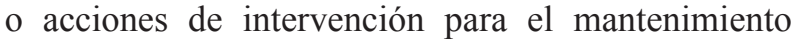

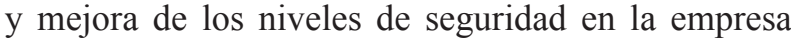

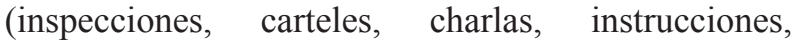

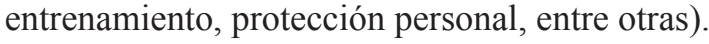

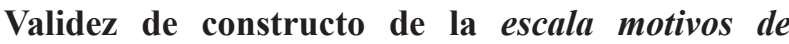

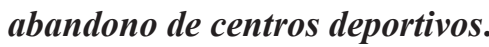

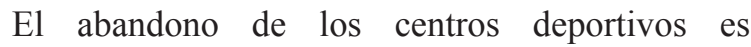

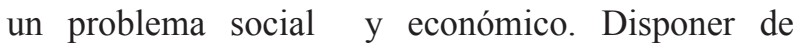

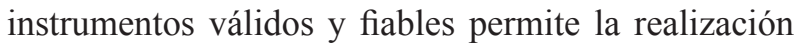

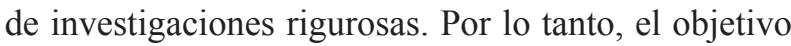

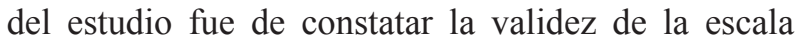

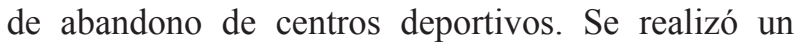

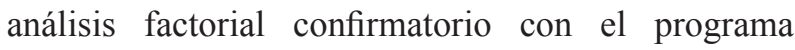


AMOS 20. Posteriormente se constató la validez discriminante mediante tres procedimientos diferentes con los programas SPSS 20 y AMOS 20: Cálculo de correlaciones entre factores, construcción de intervalos de confianza de la correlación de factores al 95\% de confianza y estimación de modelos alternativos. El resultado final fue una escala de cinco factores y doce ítems que cumple con los requisitos de validez y fiabilidad exigibles en un instrumento de investigación (Nuviala, Grao-Cruces, Teva-Villén, Pérez-Ordás y Blanco-Luengo, 2012). En este estudio la muestra la conformaron 642 personas que habían abandonado un centro deportivo de una ciudad española. De ellas, el $55,4 \%$ fueron varones y el $44,6 \%$ fueron mujeres, con una edad media $30,1 \pm 9,8$. El 79,2\% tenía estudios universitarios y solamente el 1,3\% manifestó poseer estudios primarios. La frecuencia semanal de práctica, fue para el 59,8\% de ellos, entre dos y tres veces por semana. Finalmente, el tiempo de práctica más habitual fue, entre 60 y 90 minutos para el $44,4 \%$ seguido, por entre 30 y 60 minutos, para el $25,2 \%$.

El 85,2\% de los sujetos formaron parte de la muestra (Nuviala, Grao-Cruces, Teva-Villén, Pérez-Ordás y Blanco-Luengo, 2012). El instrumento utilizado fue la escala de motivos de abandono de centros deportivos (Nuviala, Grao-Cruces, Teva-Villén, Pérez-Ordás y Blanco-Luengo, 2012). En este diseño se siguieron tres fases diferentes: 1) Búsqueda bibliográfica sobre abandono deportivo; 2) Realización de un grupo de discusión con profesionales del sector y con expertos en la construcción de instrumentos de investigación y 3) La realización de un estudio piloto con 50 clientes de servicios deportivos que habían abandonado la práctica deportivo. La escala tipo Likert, de uno (totalmente en desacuerdo) a cinco (totalmente de acuerdo), estaba compuesta por siete factores y 51 ítems que podían originar el abandono deportivo.

Para comprobar la estructura factorial de la escala conformada por cinco factores y 22 ítems, se aplicó un análisis factorial confirmatorio. El modelo inicial propuesto cumplió con los requisitos establecidos por Bollen (1989), según el cual, lo ideal para este tipo de análisis es la existencia de al menos tres ítems por factor. De la misma forma, el tamaño de la muestra supera los requisitos establecidos por diversos autores, a saber, el número mínimo estaría conformado por 200 sujetos cuando el número de ítems es de tres por variable latente e incluso superior si el número de ítems decrece por variable latente (Nuviala, GraoCruces, Teva-Villén, Pérez-Ordás y Blanco-Luengo).

\section{Objetivos}

\section{General}

Determinar la validez de constructo de la "Encuesta de Clima Organizacional de Pulido" en una muestra de colaboradores de tres empresas de Lima metropolitana.

\section{Objetivos específicos}

1. Identificar el grado de saturación del constructo clima organizacional, medida por la encuesta de clima organizacional de Pulido, con la variable cultura ad hoc

2. Identificar el grado de saturación del constructo clima organizacional, medida por la encuesta de clima organizacional de Pulido, con la variable estrategia.

3. Identificar el grado de saturación del constructo clima organizacional, medida por la encuesta de clima organizacional de Pulido", con la variable diseño.

4. Identificar el grado de saturación del constructo clima organizacional, medida por la encuesta de clima organizacional de Pulido", con la variable sistema de conocimiento.

\section{METODOLOGÍA}

De acuerdo al problema la investigación de tipo fue descriptiva, donde se aplicó un diseño psicométrico pues se utilizó un instrumento psicológico de medición, centrándose en el estudio de sus propiedades. Asimismo, según lo referido por Hernández, Fernández y Baptista (2007), se enmarca dentro del diseño de investigación "no experimental"; ya que se llevó a cabo sin manipular deliberadamente las variables.

El muestreo utilizado para la recolección de los datos fue de tipo intencional, por conglomerados (cada organización), siendo en total 1285 colaboradores los que participaron al momento de aplicar el instrumento ECOP.

El instrumento aplicado fue una encuesta que consta de 88 ítems, incluyendo la escala de filtro "L" con seis ítems (para poder detectar indicios de deshonestidad). La administración de la encuesta duró un tiempo que oscila entre 30 y 40 minutos, y puede ser aplicada de manera individual, grupal y colectiva. 


\section{RESULTADOS}

El diagrama de secuencias que se presenta no sólo ha permitido establecer relaciones predictivas entre constructos, sino también relaciones asociativas (correlaciones) entre los constructos e incluso entre los indicadores. Todos los constructos de un diagrama de secuencias puede clasificarse en dos clases: exógenos y endógenos; para el caso de esta investigación el constructo exógeno es clima organizacional y los constructos endógenos son: cultura ad hoc, estrategia, diseño y sistemas de información. Adicionalmente, se presentan en la figura 1 cuatro variaciones de error (e1, e2, e3 y e4) que son considerados constructos exógenos, pero que en este caso son desconocidos.

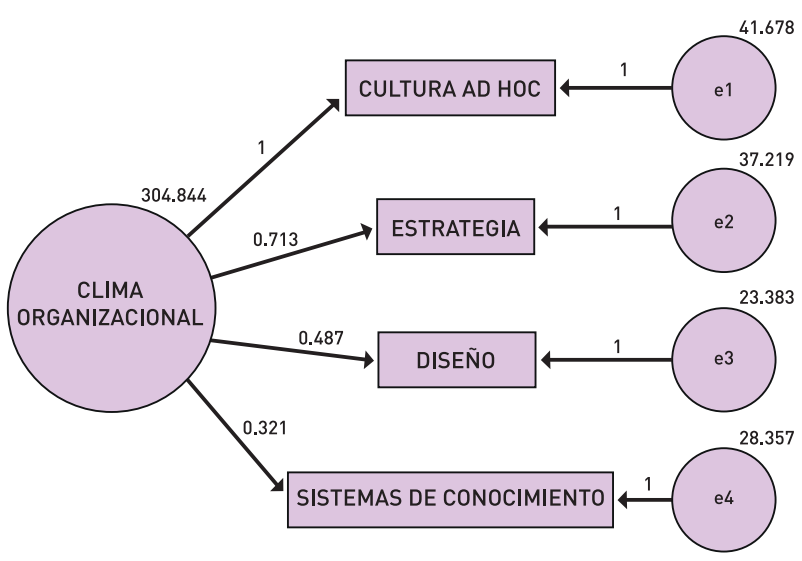

Figura 1. Diagrama de secuencias de la encuesta de clima organizacional de Pulido.
En la Tabla 1 se detallan los pesos de regresión que presenta el modelo de la encuesta de clima organizacional de Pulido. La interpretación para las estimaciones que tiene cada dimensión será: $s i$ cultura ad hoc se incrementa en 1.000 unidades, pero estrategia, diseño y sistemas de conocimiento permanecen sin cambiar, el incremento esperado para clima organizacional será de 1.000 unidades y así para cada una de las dimensiones. Cada error estándar (S.E.) es una medida de la exactitud de la estimación del parámetro y las razones críticas (C.R.) son valores t que se calculan dividiendo las estimaciones entre el error estándar (Hair, 1999). Cuando los valores de C.R. (t) son bastante altos, como en este caso, se dice que el parámetro es significativo, y que se puede tener confianza en que la variable correspondiente está asociada al constructo clima organizacional.

En la Tabla 2 se detallan las estimaciones de las correlaciones múltiples que se obtuvieron al analizar los 1285 casos de estudio de las tres empresas. Los resultados indican que el modelo explicaría el 53\% de la varianza para la dimensión sistemas de conocimiento, el $76 \%$ para diseño, el $81 \%$ para estrategia y el $88 \%$ para cultura ad hoc.

En la Tabla 3 se detallan los índices de ajuste aplicados al modelo propuesto por el autor de la encuesta de clima organizacional de Pulido. El índice de ajuste normado como medida de discrepancia entre el modelo ajustado y el modelo base (NFI: Normed

Tabla 1: Pesos de regresión (Número de grupo 1 - Modelo por defecto)

\begin{tabular}{lllcccc}
\hline \multicolumn{1}{c}{ Variables } & & \multicolumn{1}{c}{ Constructo } & Estimaciones & S.E. & C.R. & P \\
\hline Cultura ad hoc & $\beta$ & Clima organizacional & 1.000 & & & \\
Estrategia & $\beta$ & Clima organizacional & .713 & .014 & 52.523 & $* * *$ \\
Diseño & $\beta$ & Clima organizacional & .487 & .010 & 48.600 & $* * *$ \\
Sistemas de conocimiento & $B$ & Clima organizacional & .321 & .010 & 33.384 & $* * *$ \\
\hline
\end{tabular}

Tabla 2: Correlaciones múltiples (Número de grupo 1 - Modelo por defecto)

\begin{tabular}{lcc}
\hline \multicolumn{1}{r}{ Variables } & Estimaciones & Porcentajes \\
\hline Sistemas de conocimiento & .525 & $53 \%$ \\
Diseño & .756 & $76 \%$ \\
Estrategia & .806 & $81 \%$ \\
Cultura ad hoc & .880 & $88 \%$ \\
\hline
\end{tabular}

Tabla 3: Índices de ajuste al modelo

\begin{tabular}{lccc}
\hline & NFI & GFI & AGFI \\
\hline Modelo por defecto & .988 & .982 & .911 \\
\hline
\end{tabular}



debe resultar con valores superiores a 0.90 para que se concluya que el modelo presenta un ajuste aceptable y los resultados de la tabla así lo indican.

En la Tabla 4 se detallan las comunalidades asignadas inicialmente a las variables (inicial) y las comunalidades reproducidas por la solución factorial (extracción), la comunalidad de una variable es la proporción de su varianza que puede ser explicada por el modelo factorial obtenido. Estudiando las comunalidades de la extracción podemos valorar cuáles de las variables son peor explicadas por el modelo y en el caso de la tabla detallada tenemos a la variable de sistemas de conocimiento que sólo es capaz de reproducir el $67,4 \%$ de su variabilidad original. A partir de esta tabla es que podemos empezar a plantearnos si el número de factores obtenidos es suficiente para explicar todas y cada una de las variables incluidas en el análisis; como también, sí, dando por bueno el número de factores extraído, alguna de las variables incluidas podría quedar fuera del análisis.

Para los resultados de la Tabla 5, se trabajó con los indicadores de las cuatro variables que resultan ser 25 (es por ello que se listan 25 componentes), aquí se ofrece un listado de los autovalores de la matriz de varianzas-covarinazas y del porcentaje de varianza que representa cada uno de ellos. Los autovalores expresan la cantidad de la varianza total que está explicada por cada factor; y los porcentajes de varianza explicada asociados a cada factor se obtienen dividiendo su correspondiente autovalor por la suma de los autovalores (la cual coincide con el número de variables). Por defecto, se extraen tantos factores como autovalores mayores que uno tiene la matriz analizada.

Tabla 6: Matriz de componentes

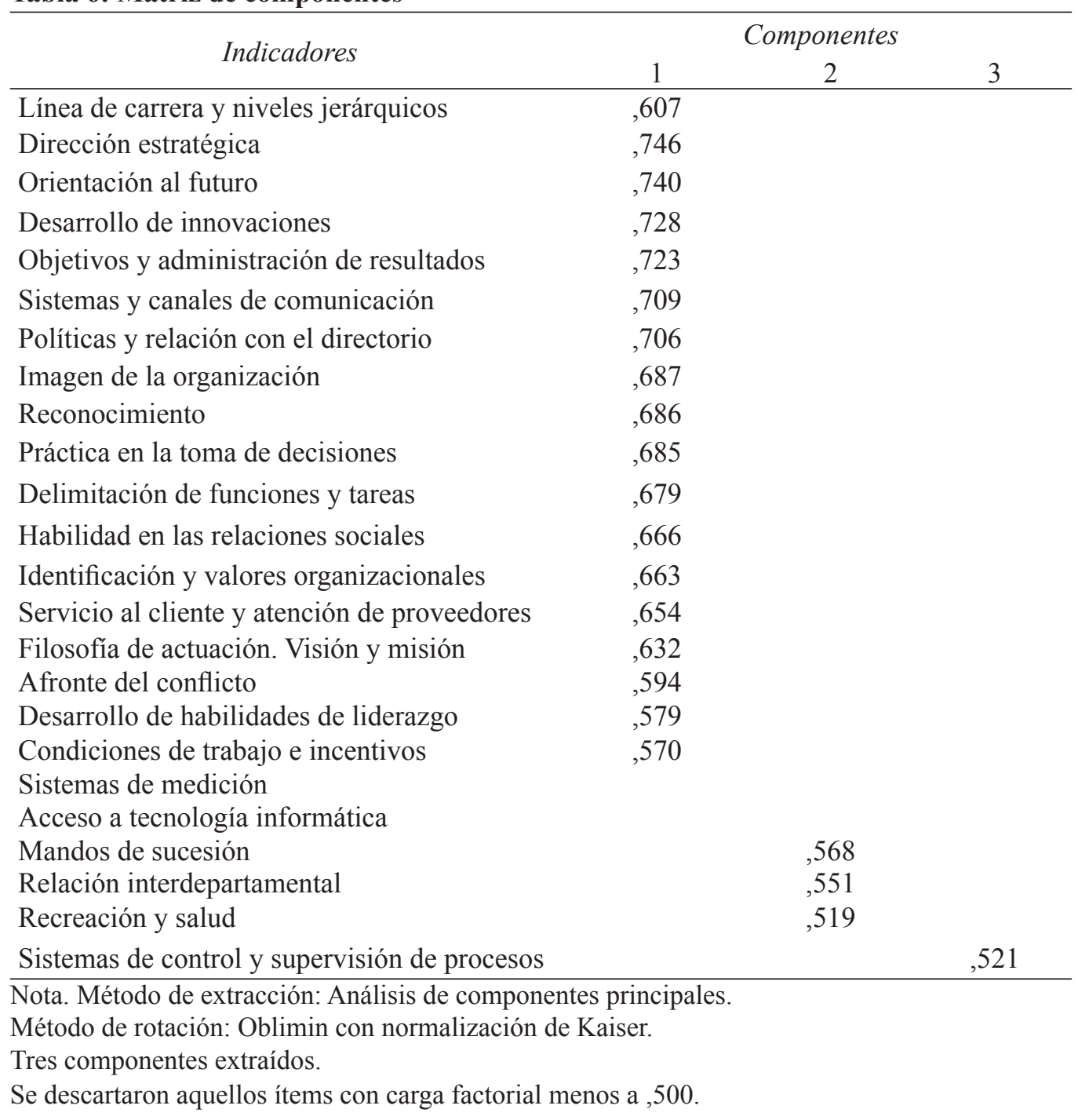



caso, el clima organizacional en colaboradores de tres empresas de Lima Metropolitana.

El principal aporte de los resultados de esta investigación radica en mejorar la encuesta de clima organizacional de Pulido -ECOP- (Pulido, 2003), al obtener un instrumento con similares propiedades psicométricas al original, pero con una reducción de ítems, considerándose finalmente sólo 72 ítems de los 82 que se tenían en el instrumento original, y sin contar la escala "L" que tiene seis ítems. Finalmente, se estaría considerando una encuesta con 78 ítems. Esta reducción podría favorecer en una aplicación más rápida y asequible para los colaboradores en las organizaciones, sin que haya una pérdida de validez (98.58\%) ni de confiabilidad (0.948) ni en la definición de su constructo hipotético, el cual intenta definir la medición objetiva del clima organizacional.

Es por ello que las consideraciones expuestas hasta aquí, remite de retorno al punto de vista según el cual la medición y validación de los constructos, en realidad, consiste en la determinación de estructuras internas y estructuras cruzadas, por lo general, en el contexto de alguna teoría más amplia que sugiere variables, constructos y sus relaciones. Los científicos no se contentan con decir sólo, qué variables particulares se relacionan entre sí; sino que tienden a hacer afirmaciones más amplias (Milájovich, 1997).

\section{CONCLUSIONES}

1. La encuesta de clima organizacional de Pulido cuenta con una correcta validez de constructo pero solo con tres factores y no con cuatro como había postulado Pulido (2003) en el análisis factorial exploratorio que aplicó al instrumento inicialmente.

2. El grado de saturación del constructo clima organizacional con la variable cultura $\mathrm{Ad} H o c$ resultó en ,874 lo que indica que dicha variable explica en un $87,4 \%$ a la encuesta de clima organizacional de Pulido" y no se eliminó ningún indicador.

3. El grado de saturación del constructo clima organizacional con la variable estrategia resultó en 0,829 que indica que dicha variable explica en un $82,9 \%$ a la encuesta de clima organizacional de Pulido y se eliminó el indicador: Desarrollo de habilidades de liderazgo con tres ítems $(35,46,48)$.

4. El grado de saturación del constructo clima organizacional con la variable diseño resultó en, 835 que indica que dicha variable explica en un
$83,5 \%$ a la encuesta de clima organizacional de Pulido y se eliminó el indicador: Condiciones de trabajo e incentivos con tres ítems $(30,36,54)$.

5. El grado de saturación del constructo clima organizacional con la variable sistemas de conocimiento resultó en ,674 que indica que dicha variable explica en un $67,4 \%$ a la encuesta de clima organizacional de Pulido y en comparación con las tres primeras sería la que menos impacto directo tiene en la encuesta o la explica menos, justamente de esta variable se eliminan dos indicadores: Sistemas de medición y relación interdepartamental con dos ítems respectivamente $(25,26)$ y $(14,31)$.

\section{REFERENCIAS BIBLIOGRÁFICAS}

1. Baptista, Fernández y Hernández, (2007). Metodología de la investigación. 4ta Edición. México: Editorial McGraw Hill.

2. González, M. \& Landero, H. (2006). Estadística con SPSS y metodología de la investigación. México: Trillas.

3. Hair, J. (1999). Análisis multivariante. 5ta Edición reimpresa. España: Editorial Pearson Educación.

4. Miljánovich, M. (1997). Validez de constructos hipotéticos en Psicología. Revista de Psicología, volumen I, número 1, julio.

5. Palma, S. (2004). Diagnóstico del clima organizacional en trabajadores dependientes de Lima Metropolitana. Lima: Universidad Ricardo Palma.

6. Pulido, C. (2003). Clima organizacional, una medida del éxito. Lima: Editorial Athanor.

7. Salaiza, F. (2008). Validez factorial de la encuesta de clima laboral de Ricardo Valenzuela. México: Universidad Virtual del Tecnológico de Monterrey

8. Tomas, U. (2012). Escala de clima social laboral - Work environment scale (WES). Recuperado 16/11/ 2014 en: http://elpsicoasesor. com/escala-de-clima-social-laboral-workenviroment-scale-wes/

9. Toro, F. (2008). Análisis psicométrico de la encuesta ECO IV de clima organizacional por países. Revista Interamericana de Psicología Organizacional, volumen 27, número 1, 44-57.

10. Venutolo, E. (2009). Estudio de clima laboral y la productividad en empresas pequeñas $y$ medianas: El transporte vertical en la ciudad autónoma de Buenos Aires (Argentina). España: Universidad Politécnica de Valencia. 